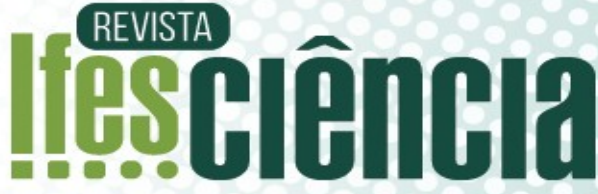

\section{SENSOR QUÍMICO DE GÁS GLP, TRANSDUÇÃO ELETRÔNICA DE SINAL E TRANSMISSÃO DE DADOS UTILIZANDO PROTOCOLO ZIGBEE}

\author{
CHEMICAL SENSOR OF LPG GAS, ELECTRONIC SIGNAL TRANS- \\ DUCTION AND DATA TRANSMISSION USING ZIGBEE PROTOCOL
}
${ }^{1 *}$ Estevão Modolo de Souza
${ }^{2}$ Júlio Nico Dantas
${ }^{3}$ Cleocir José Dalmaschio

\author{
${ }^{1}$ Instituto Federal do Espírito Santo.E-mail: estevao.souza@ifes.edu.br \\ ${ }^{2}$ Universidade Federal do Espírito Santo. E-mail: julionicodantas@gmail.com \\ ${ }^{3}$ Univerdiade Federal do Espírito Santo. E-mail: cleocir.dalmaschio@ufes.br \\ *Autor de correspondência
}

Artigo submetido em 24/07/2020, aceito em 18/12/2020 e publicado em 28/12/2020.

\begin{abstract}
Resumo: Os sensores são um dos pilares da tecnologia contemporânea. Suas aplicações são inúmeras para diferentes campos. Assim, torna-se indispensável o desenvolvimento de sensores e métodos de comunicação entre dispositivos, bem como equipamentos de controle. Este trabalho propôs um sistema para detecção em fase gasosa de gás liquefeito de petróleo (GLP) em mistura com ar, utilizando um aparato experimental formado por elemento sensor de gás, controlador de temperatura e uma placa Arduino equipada com um transmissor de dados sem fio Zigbee. O trabalho envolveu a área de química e computação para atingir desde o desenvolvimento do elemento sensor a base de $\mathrm{SnO}_{2}$ até a instrumentação, apresentando assim a preparação da suspensão coloidal, deposição e tratamento térmico do filme, a montagem do sistema de mistura de gases e sistema de aquecimento do elemento sensor, os acoplamentos do microcontrolador para transmissão de dados através de dispositivos Zigbee, a elaboração e construção do circuito eletrônico, bem como a caracterização do filme de óxido e as medidas de sensor em atmosfera de mistura gasosa envolvendo ar e GLP. Um microcomputador, utilizando um programa escrito em linguagem Python, armazenou os dados, condicionados por um microcontrolador, recebidos por meio de rede sem fio Zigbee. Além dos resultados técnicos para o sensor desenvolvido com sensibilidade dentro dos limites de inflamabilidade da mistura ar e GLP, o trabalho permite concluir que o envolvimento de áreas relacionadas permite o desenvolvimento de trabalhos amplos envolvendo desde a elaboração até a instrumentação de sensor.
\end{abstract}

Palavras-chave: instrumentação; Arduino; sensor de gás; $\mathrm{SnO}_{2}$; Zigbee. 
Abstract: Sensors, in general, are a pillar of contemporary technology. Their applications, types and models are used in several fields. Thus, it becomes indispensable the development of sensors and wireless communication methods between devices, even as equipment control. This work proposed a system to detect liquefied petroleum gas (LPG) in a mixture with air, using an experimental apparatus organized by the gas sensor element, a temperature controller and an Arduino board equipped with a Zigbee wireless data transmitter. The work involved the area of chemistry and computing to reach aims, from development of $\mathrm{SnO}_{2}$ sensor element to instrumentation. The text presents the preparation of colloidal suspension, film deposition and thermal treatment, gas mixture system assembly and sensor element heating. Also, it was described the microcontroller couplings for data transmission via Zigbee devices, the design and construction of the voltage divider circuit, as well as the characterization of the oxide film and sensor measurements in a gas and LPG atmosphere. A microcomputer, using a program written in Python, stored the data, conditioned by a microcontroller, received through the Zigbee wireless network. In addition to the technical results for the gas sensor, developed with sensitivity within the limits of flammability, the work allows to conclude that the involvement of related areas can result in the development of extensive works from sensor preparation to instrumentation.

Keywords: instrumentation; Arduino; gas sensor; $\mathrm{SnO}_{2}$; Zigbee.

\section{INTRODUÇÃO}

O grau de desenvolvimento de uma sociedade industrializada pode ser avaliado, dentre outros fatores, pelo uso dos instrumentos de medição, dispositivos capazes de detectar, medir, registrar ou controlar a variação de parâmetros em um dado processo (ADAMOWSKI; GILDER, 2004). Os dispositivos associados às tarefas de detecção que possibilitam a atuação ou registros em processos dependem do uso de sensores, que no mundo contemporâneo, são utilizados nas mais variadas aplicações: nos smartphones, nos carros, nos computadores, nos aviões, na indústria, na agricultura de precisão entre outros. A aplicação em diversas áreas demonstra que os sensores são tecnologias que foram incorporadas a sociedade e ainda experimentarão uma crescente aplicação com o avanço da internet das coisas / indústria 4.0 e as residências inteligentes e seguirá nas próximas décadas expandindo a utilização principalmente nas implementações de automação e integração da mecatrônica seja em nível residencial, seja nos modernos sistemas de automação industrial (AQUINO, 2015).

A aplicação de sensores no cotidiano envolve geralmente um conjunto eletrônico associado. A eletrônica além da automação também é responsável pela transdução de um sinal efetivamente associado a variação de uma propriedade física. Essa conversão do sinal do sensor em um sinal mensurável é feita por um transdutor, sendo que a associação de ambos possibilita identificar a variação de uma propriedade. Assim, o elemento sensor é responsável por detectar a variação de uma propriedade que pode ser física, química ou biológica e o transdutor converte essa variação da propriedade em um sinal que pode ser medido ou comparado (BALBINOT; BRUSAMARELLO, 2010).

Neste contexto, foi articulada e planejada a criação de um sistema de baixo custo para a medição da concentração gasosa em uma câmara através de um aparato experimental formado por sensor de gás, um controlador de temperatura e um microcontrolador com transmissão de dados sem fio por meio de protocolo Zigbee. O estudo teve como objetivo tanto desenvolver o elemento sensor a base de $\mathrm{SnO}_{2}$ e integrar o dispositivo a um sistema transdutor e transmissor de dados com análise em um software desenvolvido em Python. O trabalho foi fruto de uma parceria entre a Universidade Federal do Espírito Santo - UFES e o Instituto Federal do Espírito Santo- IFES por 
intermédio do programa de iniciação científica Pibic Jr. fomentado pela fundação de apoio à pesquisa do Espírito Santo - FAPES, sendo executado por alunos de graduação da área de Química e de Computação e envolveu alunos do curso eletrotécnica.

Para uma melhor compreensão por parte do leitor, o artigo foi dividido nas seguintes partes: referencial teórico relata-se os fundamentos e a implementação que tornam possível a fabricação de um elemento sensível à diferentes concentrações gasosas; em aparato experimental; em metodologia é descrito o equipamento desenvolvido para ler e interpretar os sinais provenientes do sensor, assim como são tratadas as técnicas que tornam possíveis o funcionamento adequado do equipamento construído; em resultados e discussão são exibidos os dados experimentais obtidos pelo conjunto desenvolvido ao ser submetido a diferentes concentrações gasosas em uma câmara; por fim são feitas as considerações finais sobre o trabalho.

\section{REFERENCIAL TEÓRICO}

A associação das diferentes propriedades físicas, químicas e biológicas aos elementos sensores leva a uma ampla gama de dispositivos que podem ser classificados de acordo com o tipo de detecção. Para exemplificar, podemos citar sensores: óticos, de som, de temperatura, de radiação, de gás, de fluxo entre uma variedade de dispositivos existentes atualmente, os quais se destinam a uma diversificada gama de aplicações.

Em relação aos sensores de gás baseados em óxidos semicondutores, com destaque aos dispositivos que utilizam como elemento sensor o óxido de estanho $\mathrm{SnO}_{2}$, o princípio de funcionamento se baseia na alteração da resistividade elétrica em função da mudança de grupos químicos ligados a superfície do óxido. Para sensores a base de $\mathrm{SnO}_{2}$ a temperatura de operação varia de 300 a $450{ }^{\circ} \mathrm{C}$, faixa na qual os grupos ligados a superfície podem ser substituídos de acordo com a atmosfera oxidante ou redutora presente e a concentração dos gases
(DEY, 2018). Ou seja, os elementos sensores de gás a base de óxidos semicondutores são sensores resistivos, nos quais a mudança de concentração de gás que reage com espécies químicas ligadas a superfície promove uma alteração na resistividade elétrica do elemento que pode ser transduzida e interpretada em função da composição da atmosfera. Em termos químicos, alteração na resistividade dos óxidos se associa principalmente a substituição de íons oxigênios adsorvidos a superfície dos cristais do óxido semicondutor. Esses íons de oxigênios ao manterem uma concentração de carga na superfície do cristal criam uma barreira de potencial denominada camada de depleção, dificultando o transporte de carga pelo material. Ao ser exposto a uma atmosfera com espécie que promovam reações para remoção dos íons de oxigênio na superfície do semicondutor, elétrons são liberados e injetados na camada de condução, reduzindo a barreira de potencial da camada de depleção promovendo assim uma redução na resistividade elétrica (FERNANDEZ; PEREZ, 2004).

Entre os óxidos semicondutores o $\mathrm{SnO}_{2}$ se destacou por suas características de sensibilidade sendo o material mais empregado nos dispositivos de detecção de gases em ambientes domésticos, comerciais e industriais (ADAMOWSKI; GILDER, 2004). Dispositivos comerciais que possuem elemento sensor de gás a base de dióxido de estanho podem ser facilmente encontrados em módulos disponíveis comercialmente para associação em microcontroladores diversos, sendo os mais comuns associados à plataforma Arduino (CO.LTD, [S.d.]). A integração destes dispositivos ao microcontrolador, junto com a flexibilidade proporcionada por plataforma de prototipagem programáveis, pode reduzir significativamente o custo quando comparamos com módulos comerciais direcionados para aplicações específicas (MALVINO, 1985).

\section{PROCESSOS METODOLÓGI- COS/MATERIAIS E MÉTODOS}




\subsection{OBTENÇÃO DOS ELEMENTOS SENSORES}

Filmes finos de $\mathrm{SnO}_{2}$ foram depositados sobre substratos de alumina a partir de uma suspensão aquosa do dióxido. Os substratos foram lavados, enxaguados em água destilada e por fim mergulhados em etanol anidro (Dinâmica - 99, 5\%) e secos antes de receberem a deposição da solução. Para obter uma suspensão coloidal estável do dióxido de estanho foram dispersos $2,55 \mathrm{~g}$ de $\mathrm{SnO}_{2}$ (Sigma-Aldrich - 99,9\% - 325 mesh), $250 \mu \mathrm{L}$ de dispersante $\mathrm{HOH}$ (MiracemaNuodex) e $250 \mu \mathrm{L}$ de PEG 400 (SigmaAldrich, massa molar entre $380-400 \mathrm{~g} / \mathrm{mol}$ ) em $2,50 \mathrm{~g}$ de água destilada. A suspensão aquosa, após a mistura de todos os componentes, foi levada ao banho ultrassônico (Branson - M1800) durante 30 min para que houvesse maior homogeneidade da suspensão. Após esse processo, a suspensão coloidal foi depositada em substratos de alumina, utilizando uma micropipeta para deposição da proporção de $0,30 \mu \mathrm{L}$ da suspensão $/ \mathrm{mm}^{2}$ de substrato. Após secagem, o filme formado passou por um tratamento térmico, em forno mufla (EDG 3000-3P), à $500{ }^{\circ} \mathrm{C}$ durante $1 \mathrm{~h}$, usando rampa de aquecimento de 3 ${ }^{\circ} \mathrm{C} / \mathrm{min}$ e resfriamento de $5{ }^{\circ} \mathrm{C} / \mathrm{min}$.

$\mathrm{O}$ óxido foi caracterizado quanto a estrutura cristalina em difratômetro de raiosX (Rigaku - Miniflex 600) com velocidade de varredura de $5 \%$ min com passo de $0,02^{\circ}$ e potência de geração de raios-X com tensão de $40 \mathrm{kV}$ e corrente de $15 \mathrm{~mA}$. Caracterização morfologia e de espessura do filme foi realizada em microscópio eletrônico de varredura (FEI modelo Inspect F-50). Medidas elétricas do tipo curvas de corrente versus tensão e o valor de resistência ao ser exposto a ar e mistura gasosa ar e gás liquefeito de petróleo (GLP) foram realizadas em equipamento tipo source-meter (Keithley modelo 2400).

3.2 MONTAGEM DO SISTEMA DE MISTURA DE GASES, CÂMARA DE GÁS E SISTEMA DE AQUECIMENTO DOS ELEMENTOS SENSORES
Para as medidas em atmosfera controlada com admissão de mistura gasosa foi montado um sistema de mistura com válvulas controladoras de fluxo responsáveis pela admissão de ar comprimido e gás liquefeito de petróleo. $\mathrm{O}$ ar comprimido foi admitido a partir do cilindro saindo com pressão constante a partir de regulador de pressão e manômetro e então admitida em uma válvula agulha controladora de fluxo (Swagelok modelo SS-SS2-VH) mantendo constante e igual a $200 \mathrm{ml} / \mathrm{min}$ a vazão de ar comprimido admitida na câmara de medidas. Para admissão de GLP, uma segunda válvula controladora de fluxo foi utilizada e calibrada para admitir em posições pré-fixadas do micrometro os fluxos de gás que resultassem na mistura com a concentração em porcentagem volume/volume, reportado nos gráficos, conforme estabelecido na realização do experimento. A Figura 1 apresenta esquematicamente a câmara de gás usada para as medidas. a câmara consiste em um invólucro confeccionado em chapas de alumínio com uma admissão para a mistura gasosa (indicado em verde na Figura 1) e uma saída para mistura gasosa (indicada em azul na Figura 1), ambas de 1/4 com rosca NPT usadas para fixação de conexão pneumática do tipo engate rápido. No interior da câmara foi desenvolvida uma placa aquecedora responsável pelo aquecimento do elemento sensor, representado na Figura 1 como a placa em branco/amarelo. E uma seção para conexões elétrica do sensor através de dispositivo zero insertion force (ZIF) (retângulo verde na Figura 1).

Figura 1: Representação esquemática da câmara de mistura de gás desenvolvida

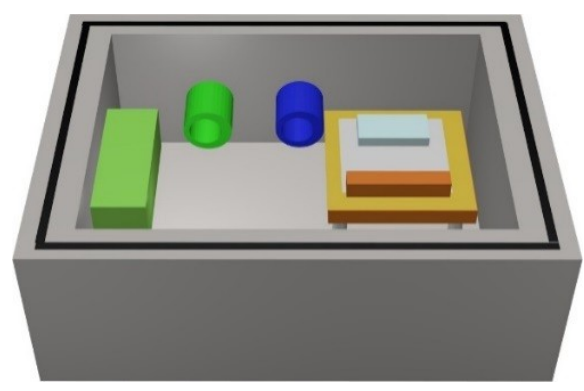

**Fonte: próprio autor. 
A segunda parte, é dividida entre o controle de temperatura, aquisição, condicionamento e transmissão do sinal do sensor. Um sensor de temperatura, termopar tipo k, posicionado abaixo do elemento sensor na placa de aquecimento, foi conectado a um controlador universal (Novus modelo N1040). Como fonte de alimentação da resistência de fio Kanthal ${ }^{\circ}$, abaixo do elemento sensor, foi utilizado uma fonte $24 \mathrm{~V}$ com corrente limite de $10 \mathrm{~A}$, sendo a potência controlada em função do tempo no controlador universal. O conjunto foi responsável por manter uma temperatura específica do filme fino de $\mathrm{SnO}_{2}$ a ser utilizado como elemento sensor de gás.

$\mathrm{Na}$ aquisição e condicionamento de sinal foram utilizados um microcontrolador Arduino Uno (AU) em conjunto com um filtro eletrônico disposto em um circuito divisor de tensão. O uso do microcontrolador justifica-se pela facilidade de tratar e automatizar o processo de medição. Para a transmissão do sinal, optou-se em fazê-la sem fio tendo em vista que em processos industriais ou comerciais, é comum o elemento sensor não estar próximo à unidade de controle (BEGA, 2006). Neste trabalho, o sinal do sensor, medido pelo Arduino, foi enviado para um computador utilizando a tecnologia Zigbee, o qual destaca-se, na indústria, por conta do seu baixo consumo de energia e a possibilidade de ser ligado em rede do tipo malha (ZIGBEE, [S.d.]).

Tanto o filtro quanto os módulos de transmissão sem fio foram conectados ao AU por meio de Shields. Estas são placas que podem ser conectadas na parte superior do Arduino, estendendo seus recursos. O Shield divisor de tensão, com circuito representado em forma de diagrama na

Figura 2, foi fabricado utilizando os métodos clássicos de simulação, desenho esquemático por software, impressão a laser em papel transfer, passagem do impresso para placa cobreada de fenolite e revelação por corrosão com solução de percloreto de ferro $\left(\mathrm{FeCl}_{3}\right)$. Na placa foram fixados pinos para se prender ao Arduino, terminais de alimentação e um capacitor para estabilizar o sinal (Filtro analógico). Um dos terminais do potenciômetro foi conectado ao pino A0 do microcontrolador. A Figura 3 representa o digrama eletrônico da Shield do circuito divisor de tensão, indicando os componentes potenciômetro e capacitor, bem como o elemento sensor.

Figura 2 - Representação do circuito contendo a Shield divisor de tensão

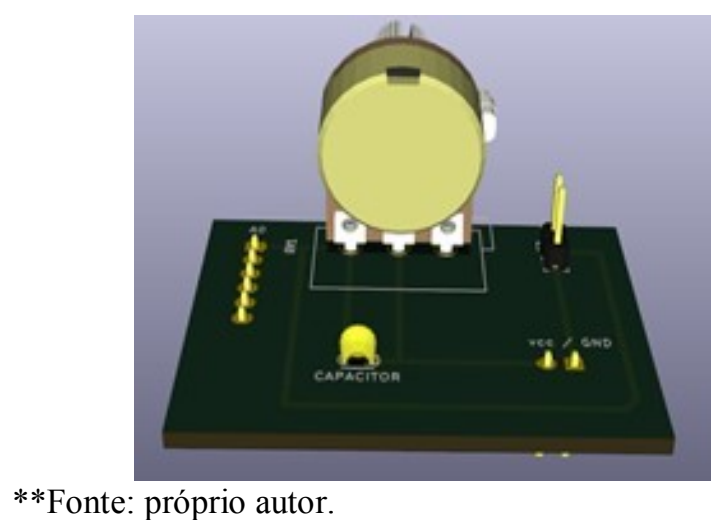

Figura 3 - Diagrama do circuito divisor de tensão

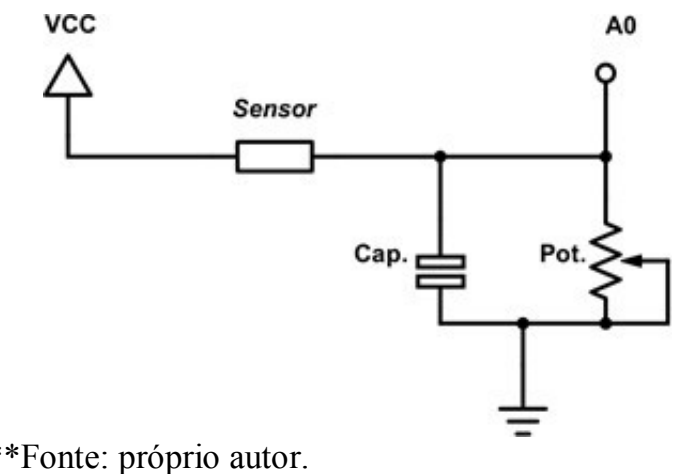

O Shield Xbee, representado na Figura 4, possibilita ao Arduino a comunicação sem fio através do protocolo Zigbee pelo acoplamento de um dispositivo de transmissão de sinal.

Figura 4 - Shield Zigbee para transmissão de dados sem fio 


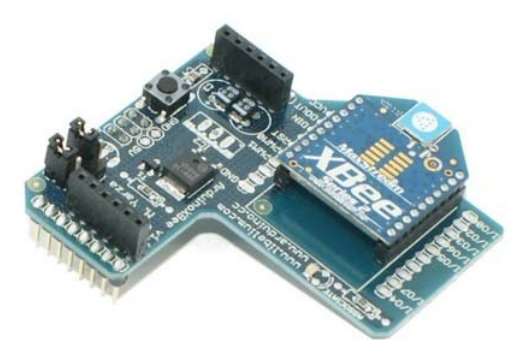

**Fonte:

http://antipastohw.pbworks.com/w/page/26626243/A rduino $\% 20 X B e e \% 20$ Shield.

\subsection{MEDIDA DE TENSÃO}

O aparato, descrito na Seção 3, permite determinar a concentração de gás confinado em um ambiente. Normalmente, as grandezas físicas não podem ser determinadas diretamente. Neste caso, a resistividade do elemento sensor é proporcional a concentração (percentual) do gás analito, assim, é possível utilizar esta propriedade para obter uma relação entre a resistência do elemento sensor com a quantidade de gás presente.

Utilizando o circuito divisor de tensão, a resistividade também pode ser medida indiretamente ao medir a tensão elétrica sobre um resistor em série com o sensor (Figura 3). A concentração gasosa, por fim, é obtida combinando-se as duas medidas indiretas. Para que isso ocorra de maneira satisfatória, o sensor deve inicialmente ser calibrado. A calibração pode ser feita por uma curva que relaciona os valores de tensão medidos com as concentrações conhecidas injetadas na câmara de gás.

A medida de tensão pelo $\mathrm{AU}$ é feita pelo conversor analógico digital (CAD). Tensões entre 0 e $5 \mathrm{~V}$ são convertidas para valores digitais entre 0 e 1023 . Observando a Figura 3, a tensão na entrada do CAD pode ser determinada como:

$$
V_{A 0}=V_{c c}\left(\frac{R_{\text {pot }}}{R_{\text {pot }}+R_{\text {sensor }}}\right)
$$

onde $\mathrm{V}_{\mathrm{A} 0}$ é a tensão na entrada do $\mathrm{CAD}, \mathrm{V}_{\mathrm{cc}}$ é a tensão que alimenta o circuito, $\mathrm{R}_{\text {pot }}$ é a resistência pré-ajustada no potenciômetro e $\mathrm{R}_{\text {sensor }}$ é a resistência do sensor.
Sendo o GLP uma mistura de gases que se comportam como agente redutor (provoca processo de oxidação ao reagir com o oxigênio adsorvido no semicondutor) e o $\mathrm{SnO}_{2}$ um semicondutor tipo n, à medida que aumenta a concentração de gás presente na câmara, a resistência do elemento sensor diminui, portanto a tensão medida no CAD aumenta. Por meio da equação anterior é possível mostrar que se obtêm uma melhor resolução ajustando a resistência $R_{\text {pot }}$ para coincidir com $\mathrm{R}_{\text {sensor }}$ quando há apenas os gases da atmosfera (ar) dentro da câmara.

\subsection{FILTRAGEM DO SINAL DE TENÃO}

Todo circuito eletrônico é sujeito a receber interferências exógenas, também conhecidas como ruídos, que atrapalham o seu funcionamento adequado (SEDRA et al., 2016). O sinal, coletado pelo CAD, é sensível a esse tipo de influência, afetando assim, a medida de concentração na mistura gasosa, no caso. Uma maneira de minimizar os efeitos dos ruídos é a utilização de filtros. Neste trabalho, foram utilizados dois tipos de filtros: um analógico e outro digital. O primeiro, consiste na instalação de um capacitor disposto de forma paralela ao sinal medido (Figura 3). Já o segundo, funciona manipulando o dado na saída do conversor $\mathrm{A} / \mathrm{D}$.

Um possível filtro digital consiste em fazer a média dos últimos valores lidos. Neste trabalho optou-se por utilizar um filtro do tipo média móvel, em que os dados vão sendo armazenados em um vetor com uma estrutura de fila. A cada novo valor armazenado, o mais antigo é descartado. A média é obtida somando todos os valores do vetor divididos pela quantidade de elementos armazenados (MITRA; KUO, 2006).

O filtro analógico é configurado ao mudar o valor da capacitância. Neste trabalho, utilizou-se um capacitor de poliéster de $82 \mathrm{nF}$. O ajuste do filtro digital se dá pelo tamanho do vetor. Se mal ajustados, ou os ruídos não serão filtrados, ou as respostas tornam-se lentas não acompanhando a variação do sinal medido (SCHAUMANN; 
MAC ELWYN VAN VALKENBURG; XIAO, 2001).

\subsection{TRANSMISSÃO DE DADOS SEM FIO}

O Zigbee permite ser configurado em dois modos: transparente e API. Para o segundo, os dados são enviados serialmente em uma estrutura, Figura 5, contendo o valor medido, o endereço de destino, dentre outros.

Figura 5 - Estrutura de dado modo API.
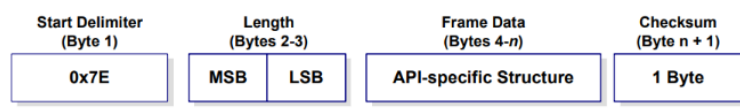

**Fonte:

https://www.digi.com/resources/documentation/digid ocs/PDFs/90000976.pdf

Acesso em : 22 de jul. de 2020.

Para o projeto foram utilizados dois módulos no modo API: um configurado como coordenador, ligado ao computador, e outro como roteador, ligado ao Arduino.

\subsection{REGISTRO E IMPRESSÃO DOS DA- DOS}

Os dados recebidos pelo Zigbee coordenador eram repassados ao computador pela porta serial. Um programa desenvolvido em Python recebia estas informações as convertendo para valores de tensão. Os valores foram armazenados, em formato de tabela, junto com o tempo em que a medida era realizada. Finalizando a medição, o programa plotava o gráfico de tensão $\mathrm{x}$ tempo.

\section{RESULTADOS E DISCUSSÃO}

\subsection{CARACTERIZAÇÃO DO $\mathrm{SnO}_{2}$ E DE- POSIÇÃO DO FILME}

$\mathrm{O} \mathrm{SnO}_{2}$ empregado para deposição dos filmes foi caracterizado quanto a estrutura cristalina, sendo identificado como estrutura cassiterita conforme ficha cristalográfica PDF \#41-1445. Na Figura 6 está apresentado o difratograma de raios-X do material empregado na deposição dos filmes, após tratamento térmico a $500^{\circ} \mathrm{C}$, mesma temperatura de tratamento do filme. $\mathrm{O}$ material apresenta estrutura cristalina bem formada conforme pode ser avaliado pelos picos intensos e bem definidos, além disso não se observa indício de material amorfo no difratograma.

Figura 6 - Difratograma de raios- $\mathrm{X}$ do material $\mathrm{SnO}_{2}$ comercial após tratamento térmico a $500^{\circ} \mathrm{C}$.

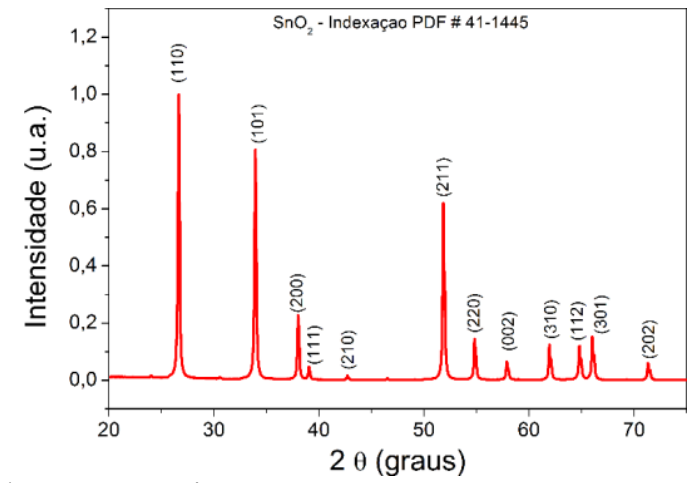

**Fonte: próprio autor.

Para deposição dos filmes foi preparado uma suspensão coloidal do óxido de estanho. A suspensão apresentou estabilidade para deposição na forma de filmes. A Figura 7 apresenta o frasco contendo a suspensão 5 minutos após cessar o uso de ultrassom. A deposição sobre substratos de alumina resulta em um filme fino, que após tratamento térmico foi utilizado como elemento sensor. A Figura 8 apresenta uma fotografia do elemento sensor (filmes + substratos) e os dois contatos utilizados para conexão do circuito elétrico.

Figura 7 - Suspensão coloidal estável após 5 min de cessar o uso de ultrassom utilizada para deposição do filme.

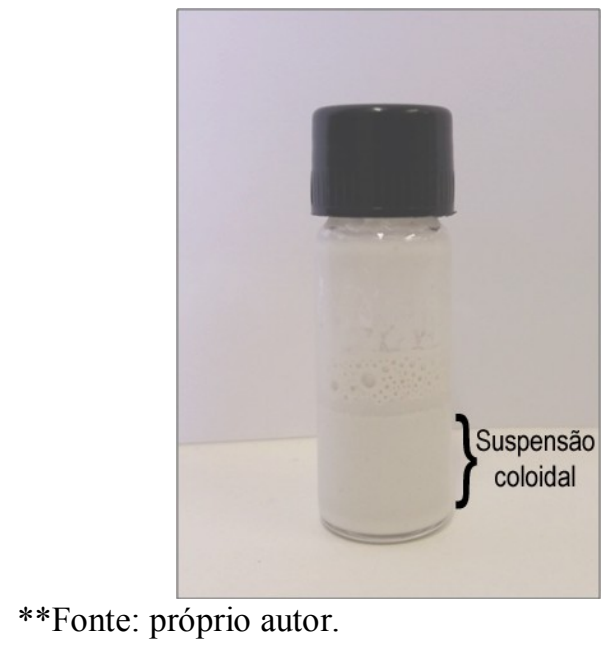


Figura 8 - Filme fino de $\mathrm{SnO}_{2}$ depositado sobre substrato de alumina e com os contatos elétricos.

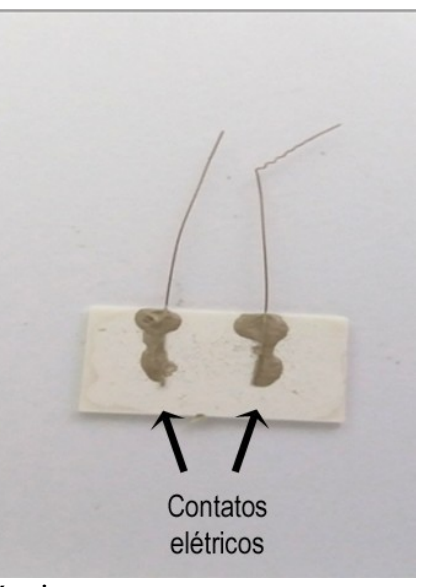

**Fonte: próprio autor.

$\mathrm{O}$ filme de $\mathrm{SnO}_{2}$ foi caracterizado quanto a microestrutura e espessura através de microscopia eletrônica de varredura, conforme apresentado na Figura 9. A imagem de topo do filme apresenta a estrutura típica de partículas aglomeradas com poros nos quais gases podem ter acesso ao interior da estrutura. A agregação tem um papel fundamental no elemento sensor, pois o percurso eletrônico terá como limites de barreira o contato entre as partículas do $\mathrm{SnO}_{2}$. O filme apresenta espessura média de $1,5 \mu \mathrm{m}$, com pouca irregularidade de espessura ao longo do perfil da fratura conforme pode ser observado na Figura 10.

Figura 9 - Microscopia eletrônica de varredura do filme de $\mathrm{SnO}_{2}$, imagem de topo do filme depositado.

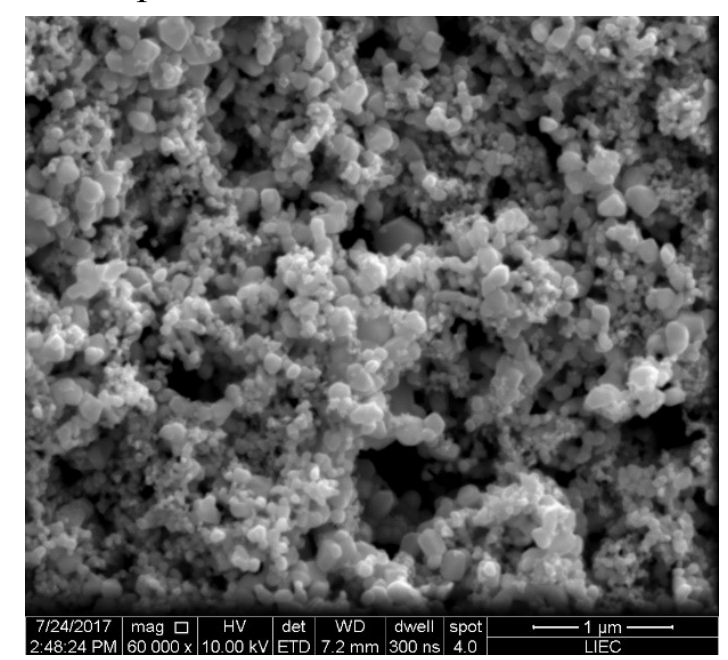

**Fonte: próprio autor.
Figura 10 - Microscopia eletrônica de varredura do filme de $\mathrm{SnO}_{2}$, imagem de seção transversal de filme fraturado para estimar a espessura.

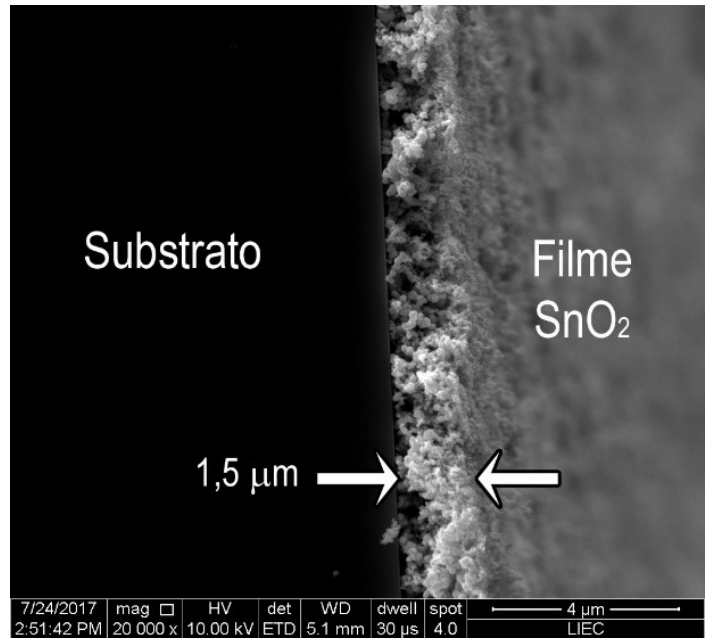

**Fonte: próprio autor.

O Filme foi caracterizado quanto ao comportamento semicondutor e o valor de resistência elétrica através de curvas de corrente tensão ajustadas segundo a lei de Omh. A Figura 11 apresenta as curvas para três temperaturas em condição de atmosfera estática de ar. Os valores de resistência ajustados estão apresentados na Tabela 1 indicando o caráter semicondutor do filme com redução da resistência ao elevar a temperatura.

Figura 11 - curvas de corrente tensão do filme de $\mathrm{SnO}_{2}$ evidenciando o caráter semicondutor do material.

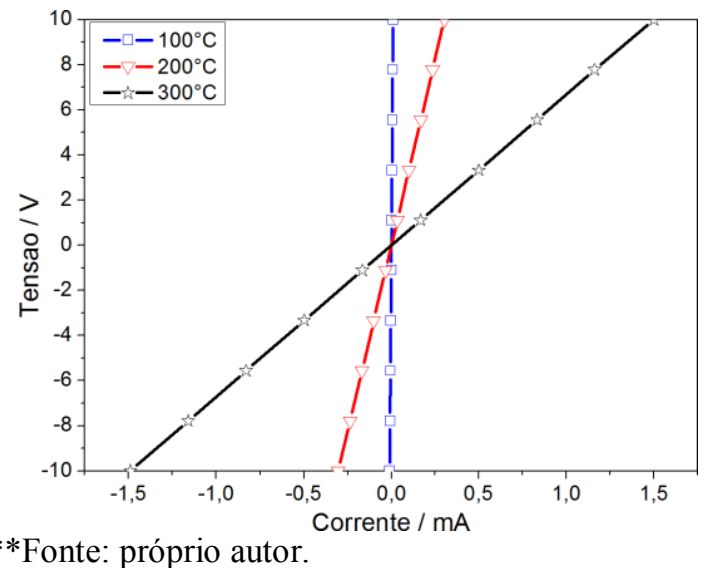

Tabela 1 - Valores de resistência nas diferentes temperaturas obtidos pelo ajuste linear segundo a lei de Ohm.

\begin{tabular}{|l|l|l}
\hline Temperatura & Resistência & Erro na
\end{tabular}




\begin{tabular}{|c|c|c|}
\hline$\left({ }^{\circ} \mathrm{C}\right)$ & $(\Omega)$ & $\begin{array}{c}\text { Resistência } \\
(\Omega)\end{array}$ \\
\hline 100 & $1,033 M$ & 232,8 \\
\hline 200 & $33,07 \mathrm{~K}$ & 16,96 \\
\hline 300 & $6,693 \mathrm{~K}$ & 5,512 \\
\hline
\end{tabular}

**Fonte: próprio autor.

Para avaliação da sensibilidade do filme ele foi analisado quanto o valor da resistência ao ser submetido a tensão de 5 volts em atmosfera com diferentes concentrações de gás GLP. O valor de resistência altera de acordo com composição da atmosfera conforme pode ser constatado na Figura 12. O sinal (S) foi avaliado com base no valor de resistência de acordo com a expressão $S=(\operatorname{Rg}-\operatorname{Rar}) / \operatorname{Rar}$, sendo $\mathrm{Rg}-$ valor de resistência na atmosfera com mistura gasosa (ar+GLP) e Rar - valor da resistência na atmosfera de ar. Os valores estão indicados no gráfico da Figura 13, no qual se observa um maior valor do sinal medido com o aumento da concentração de gás. Os valores reportados na avaliação se referem a faixa de concentração 3 a $35 \%$ em volume de GLP na mistura gasosa. Indicando que o dispositivo tem potencial de utilização em misturas dentro do limite de inflamabilidade (limites de explosividade) da mistura ar e GLP, sendo estes limites para a mistura mencionada como limite inferior de 1,81 e limite superior de 8,86\% (MISHRA; RAHMAN, 2003).

Figura 12 - Avaliação do comportamento sensor do elemento frente mistura gasosa ar e GLP. Curva de resistência em função da concentração de gás avaliada em $300^{\circ} \mathrm{C}$.

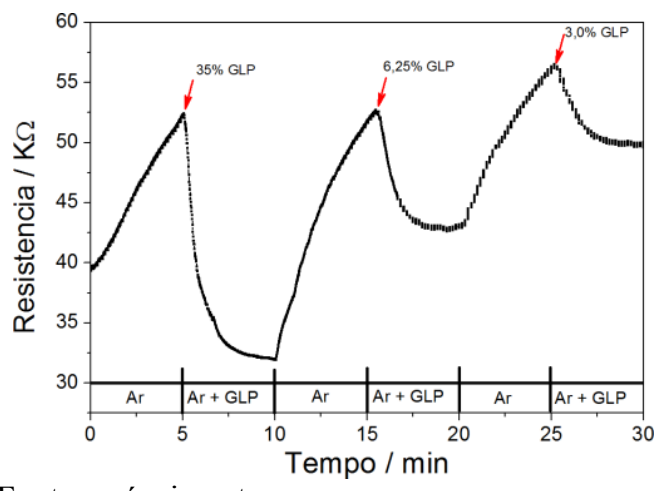

**Fonte: próprio autor.
Figura 13 - Sinal elétrico com base na variação de resistência elétrica no filme de $\mathrm{SnO} 2$ submetido a mistura gasosa GLP e ar.

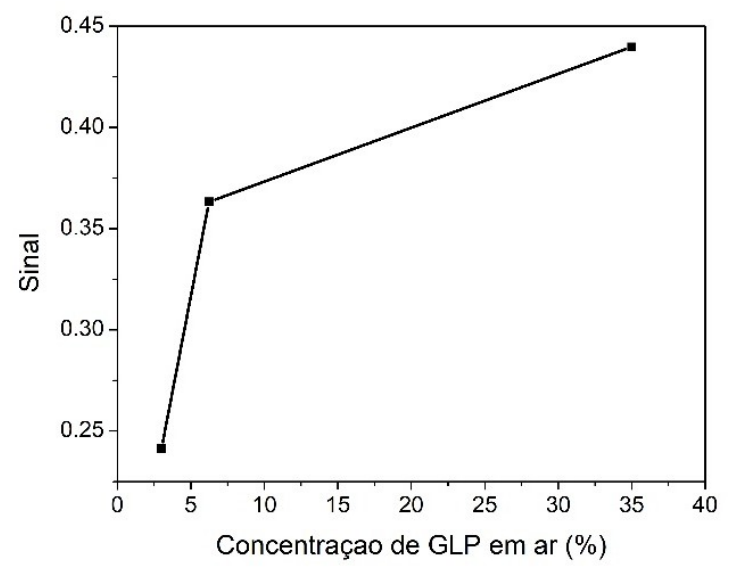

**Fonte: próprio autor.

\subsection{DERMINAÇÃO DA CONCENTRA- ÇÃO DE GÁS EMPREGANDO CIRCUI- TO DESENVOLVIDO COM MICRO- CONTROLADOR E CIRCUITO DIVISOR DE TENSÃO}

Para que a concentração de gás inserida na câmara, Figura 14 pudesse ser medida de acordo com a metodologia relatada na Seção 3, foi desenvolvido o circuito da Figura 15 contendo o microcontrolador, o circuito divisor de tensão e o transmissor de dados Zigbee. Os dados obtidos através do sistema de medidas descrito e utilizando o elemento sensor a base de $\mathrm{SnO}_{2}$ podem ser vistos na Tabela 2 que constam alguns pontos não sequenciais referentes à

Figura 16. Nesta Figura, as setas em vermelho destacam o momento em que diferentes concentrações de gás foram injetadas na câmara. Os respectivos valores de tensão foram utilizados para a calibração do sensor.

Por fim, todos os dados eram armazenados em arquivo csv e os gráficos plotados armazenados na forma de imagem. A vantagem do formato csv é que este permite a utilização dos dados em programas de planilha eletrônica, além do formato usado no trabalho. 
Figura 14 - Câmara de gás construída no trabalho

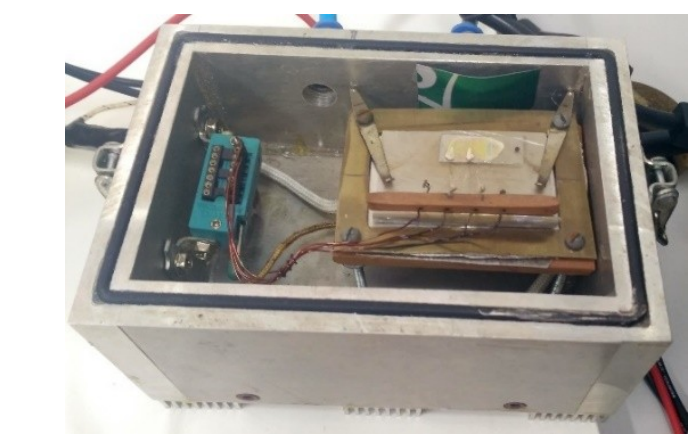

**Fonte: próprio autor.

Figura 15 - Montagem envolvendo microcontrolador, Shield Zigbee e Shield divisor de tensão

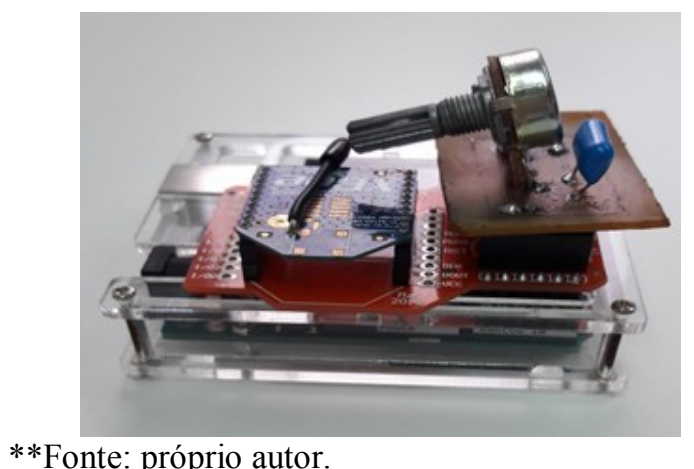

**Fonte: próprio autor.

Figura 16 - Tensão na saída do divisor de tensão proporcional à concentração de gás no interior da câmara - medida realizada a $350^{\circ} \mathrm{C}$.

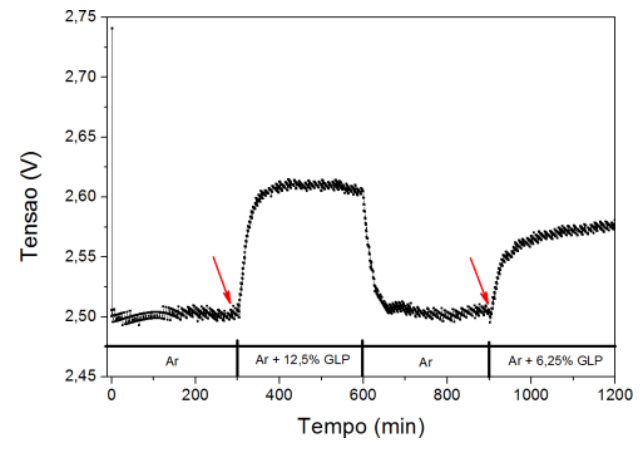

**Fonte: próprio autor.

Tabela 2 - Valores de tensão proporcionais à concentração de gás medidos em diferentes tempos.

\begin{tabular}{|l|l|}
\hline Tensão & Tempo \\
\hline
\end{tabular}

\begin{tabular}{|c|c|}
\hline$(\mathrm{V})$ & $(\mathrm{s})$ \\
\hline 2,52 & 0 \\
\hline 2,58 & 311 \\
\hline 2,72 & 457 \\
\hline 2,78 & 825 \\
\hline 2,85 & 926 \\
\hline
\end{tabular}

Fonte: próprio autor.

\section{CONCLUSÕES}

Em relação ao desenvolvimento técnico do sensor e sistema de medidas e transmissão de dados se constata que o elemento sensor possuí limites de sensibilidade com potencial utilização dentro dos limites de inflamabilidade da mistura gasosa ar e GLP. Sendo assim, o processo de preparação da suspensão, deposição e tratamento térmico do filme de $\mathrm{SnO}_{2}$ utilizado se apresentou adequado ao desenvolvimento de sensores de gás a base deste material.

Para o desenvolvimento instrumental, leitura do sensor, o tratamento e transmissão do sinal por tecnologia Zigbee, pode ser facilmente adaptada para outros sensores de naturezas distintas ou qualquer aplicação que tenha como saída uma tensão elétrica. Sendo que no conjunto da implementação eletrônica, cuidados tiveram que ser tomados para evitar que os ruídos impossibilitassem a medida correta que se propõem. Os filtros capacitivo e digital implementados apresentaram respostas (valores de tensão elétrica) adequada, tal que os resultados obtidos a partir do conjunto construído com a plataforma de prototipagem e o circuito divisor de tensão permite se compara as medidas a dados obtidos em multímetro de bancada comercial.

Por conta do projeto ser de um programa de incentivo à pesquisa com alunos de ensino médio, cuidados tiveram que ser adotados na escolha das linguagens de programação. Tanto a linguagem própria do Arduino quanto o Python possuem documentação extensa e pode ser encontrada com facilidade na internet as quais ajudaram 
na implementação do projeto. Além disso para a realização das etapas, duas frentes de trabalho foram criadas: uma responsável pela criação do sensor e a outra pela instrumentação, as quais culminaram em conjunto possibilitaram a construção de um instrumento medidor de concentração gasosa de GLP em ar.

\section{AGRADECIMENTOS}

Os autores agradecem as agências de fomentos: CNPq e FAPES - Processo 71907980 .

\section{REFERÊNCIAS}

ADAMOWSKI, J C; GILDER, N.

Sensores: Tecnologias e Aplicações. .

[S.1.]: AlphaMidia Assessoria Fonografica, São Paulo. , 2004

AQUINO, André. Sensores conectados em rede. Revista da Sociedade Brasileira de Computação, v. 29, n. 4, p. 9-13, 2015.

BALBINOT, Alexandre;

BRUSAMARELLO, Valner João.

Instrumentação e fundamentos de medidas--vol. 1. Livros Técnico E Científicos Editora, 2010.

BEGA, Egidio Alberto. Instrumentação industrial. [S.1.]: Interciência, 2006.

CO.LTD, H. E. Technical data MQ-3 gas sensor. Disponível em:

$<$ https://www.sparkfun.com/datasheets/Sens ors/MQ-3.pdf>.

Acesso em: 05 jun. 2020.

DEY, Ananya. Semiconductor metal oxide gas sensors: A review. Materials Science and Engineering: B, v. 229, p. 206-217, 2018 .

FERNANDEZ, F.X.R.; PEREZ, H.E.M. . Sensores de gás. 1. ed. [S.1.]: USP, 2004. .

MALVINO, A. P. Microcontroladores e microprocessadores. São Paulo: [s.n.],
1985.

MISHRA, D P; RAHMAN, A. An experimental study of flammability limits of LPG/air mixtures. Fuel, v. 82, n. 7, p. 863866, 2003.

MITRA, Sanjit Kumar; KUO, Yonghong. Digital signal processing: a computerbased approach. [S.1.]: McGraw-Hill New York, 2006. v. 2.

SCHAUMANN, Rolf; MAC ELWYN VAN VALKENBURG, Xiao; XIAO, Haiqiao. Design of analog filters. [S.1.]: Oxford University Press New York, 2001. v. 1.

SEDRA, Adel S et al. Microelectronic circuits. [S.1.]: Oxford University Press, 2016.

ZIGBEE, A. Zigbee 2006 specification. Disponível em: $<$ https://www.zigbee.org/>. Acesso em: 10 jul. 2020. 